\title{
Characterization of a rolling-circle replication plasmid from Thermus aquaticus NTU103
}

\author{
Sheng-Fen Chu ${ }^{\mathrm{a}, 1}$, Hung-Yu Shu ${ }^{\mathrm{b}}$, Ling-Chun Lin ${ }^{\mathrm{a}}$, Mao-Yen Chen ${ }^{\mathrm{c}}$, \\ San-San Tsay ${ }^{\mathrm{d}}$, Guang-Huey Lin ${ }^{\mathrm{a}, *}$ \\ ${ }^{a}$ Microbial Genetics Laboratory, Institute of Microbiology, Immunology and Molecular Medicine, Tzu-Chi University, Hualien 970, Taiwan \\ ${ }^{\mathrm{b}}$ Sequencing Core, Genome Research Center, National Yang Ming University, Taipei 112, Taiwan \\ ${ }^{\mathrm{c}}$ Institute of Biological Chemistry, Academic Sinica, Taipei 115, Taiwan \\ ${ }^{\mathrm{d}}$ Institute of Plant Biology, National Taiwan University, Taipei 106, Taiwan
}

Received 13 October 2005, revised 25 January 2006

Available online 3 May 2006

Communicated by Rolf Bernander

\begin{abstract}
The thermophilic bacterium Thermus aquaticus NTU103 harbors a 1965-bp plasmid, pTA103. Sequencing analysis revealed that pTA103 contains two open reading frames. One of the open reading frames (orf2) shares no significant homology with protein in the data bank. The other one has $50 \%$ similarity and $34 \%$ identity with RepA-like protein of $\mathrm{pRm} 1132 \mathrm{f}$, which is a rolling-circle replication (RCR) plasmid isolated from Sinorhizobium meliloti. S1 nuclease analysis demonstrated that pTA103 contains a single-stranded intermediate, confirming that pTA103 replicates via RCR mechanism. Sequence data also revealed putative double-stranded origin and single-stranded origin sites, indicating the importance of these cis elements in pTA103 replication.
\end{abstract}

(C) 2006 Elsevier Inc. All rights reserved.

Keywords: Rolling-circle replication plasmid; pTA103; Thermus aquaticus

\section{Introduction}

Thermus aquaticus is a gram-negative thermophilic bacillus that produces $T a q$ polymerase, which is a powerful tool for the polymerase chain reaction (Brock and Freeze, 1969). Many Thermus spp. contain plasmids whose function has not been studied thor-

\footnotetext{
${ }^{*}$ Corresponding author. Fax: +886 38566724.

E-mail address: veronica@mail.tcu.edu.tw (G.-H. Lin).

1 Present address: Mackay Memorial Hospital, 92, Section 2, Chungshan North Road, Taipei, Taiwan.
}

oughly. Although most of these plasmids are cryptic, a large plasmid of Thermus thermophilius has been demonstrated to contain a gene cluster encoding $\beta$-carotene (Tabata et al., 1994). Mather and Fee (1990) also reported that plasmid pVV8 is associated with coaggregation in T. thermophilus HB8. Meanwhile, no bacteriocin production, antibiotics resistance or metal ion-binding capabilities has been found to be associated with those plasmids (Hishinuma et al., 1978; Vásquez et al., 1983). Most plasmids have been isolated and served as shuttle vectors for gene expression or protein engineering from thermophilic bacteria 
(Jonuscheit et al., 2003; Lasa et al., 1992; Wayne and $\mathrm{Xu}, 1997)$. Few investigations have focused on the replication mechanism of these plasmids. The first characterized plasmid for revealing the replicative mechanism of thermophilic plasmids is pRQ7, which is present on the hyperthemophilic bacterium Thermotoga species strain RQ7. Plasmid pRQ7 was identified as a rolling-circle-replicating (RCR $)^{2}$ plasmid and was the smallest plasmid described so far (Harriott et al., 1994; Yu and Noll, 1997).

Rolling-circle-replicating plasmids are omnipresent in Gram-positive bacteria (del Solar et al., 1998; Khan, 1997, 2005). More plasmids from gram-negative bacteria have been found to replicate by the same replication mechanism (Gruss and Ehrlich, 1989; Kleanthous et al., 1991; Kues and Stahl, 1989; Yasukawa et al., 1991). More than 200 RCR plasmids have been described and these plasmids can be separated into many families based on sequence homologies of the initiation protein (Rep-like protein) and double-stranded origin (dso) (Khan, 1997, 2005). RCR plasmid was initially isolated from Staphylococccus aureus and was present in numerous gram-positive bacteria including Bacillus subtilis, Streptococcus agalactiae, Clostridum butyricum, Lactococcus lactis, and Streptomyces. Most plasmids in gram-negative bacteria replicated by theta replication. However, plasmids isolated from Helicobater pylori, Selnomonas ruminantium, and Shigella sonnei also have found involving RCR mechanism (Khan, 1997). Erauso et al. (1996) also reported a plasmid, pGT5, in hyperthermophilic archaeon Pyrococccus abyssi replicated by the RCR mechanism.

Rolling-circle-replicating plasmids replicated by plasmid-encoded replication initiation proteins (Rep), which bind to the $d s o$, nick the positive (leading) strand of the plasmid DNA before the leading strand is synthesized. The lagging strand is synthesized from the single-stranded origin (sso) after a single-stranded intermediate was released from the double-stranded plasmid (del Solar et al., 1998; Zaman et al., 1993). Hence, the Rep, dso, and sso are the critical elements for RCR replication mechanisms (Khan, 1997, 2005).

This investigation sequenced pTA103, which is a plasmid isolated from T. aquaticus NTU103. Based on our complete sequence analysis, pTA103 contains a rep-like gene and one unknown open reading

\footnotetext{
${ }^{2}$ Abbreviations used: RCR, rolling-circle-replicating; dso, double-stranded origin; sso, single-stranded origin; Rep, replication initiation protein.
}

frame transcribed from the opposite direction. S1 nuclease analysis shows that pTA103 replicated via a single-stranded intermediate which is the direct evidence of RCR. A putative $d s o$ and sso downstream of the rep-like gene also were proposed.

\section{Materials and methods}

\subsection{Bacterial, plasmid preparation, and medium}

Thermus aquaticus NTU103, a isolate from a hot spring in Taiwan, was the host strain of pTA103. T. aquaticus YT-1 (ATCC25104) was obtained from America Type Culture Collections. Plasmids from these strains were prepared by the method of Kado and Liu (1981) and modified alkaline lysis method of Birnboim and Doly (1979). Plasmid pTA103 and the smaller plasmids of T. aquaticus YT-1 were purified by the standard alkaline extraction procedure except that the clear lysate was vortexed vigorously for $30 \mathrm{~s}$ to destroy the large plasmids. Thermus medium was prepared by the method of Williams and da Costa with mild modification (1992). T. aquaticus NTU103 was cultured on Thermus agar plates, which were prepared with $20-25 \mathrm{~g} / \mathrm{L}$ agar. The plates were sealed in plastic bags and incubated at $60^{\circ} \mathrm{C}$. Escherichia coli strain DH5 $\alpha$ was used for gene manipulation.

\subsection{In vitro transposition and $D N A$ sequencing}

EN::TN $\langle$ R6K ori KAN-2〉 (Epicentre; Madison, WI) was inserted into pTA103 by in vitro transposition (Goryshin and Reznikoff, 1998; York et al., 1998). Plasmids that contained an EN::TN $\langle\mathrm{R} 6 \mathrm{~K}$ rori $\mathrm{KAN}-2\rangle$ insertion were selected on LB-kanamycin plates after transforming the plasmids into TransforMax EC100D pir $^{+}$electrocompetent $E$. coli (Epicentre). DNA was sequenced with KAN-2 FP-1 forward primer (5'-ACCTACAACAAAGCTCTCA TCAACC) and R6KKan-2 RP-1 reverse primer (5'-C TACCCTGTGGAACACCTACATCT) near the borders of the transposon. 16S rRNA gene of NTU 103 was amplified with 16S rRNA gene conserved primers $27 \mathrm{f}$ (5'-AGAGTTTGATCCTGGCTCAG) and 1522r (5'-AA GGAGGTGATCCA(AG)CCGCA). After amplification, 16S rRNA gene was cloned into pGEM-7zf(-) (Promega) for full-length sequence analysis by M13 forward and reverse universal primers.

\subsection{Analyzing the rep and orf2 transcripts}

Total RNA was isolated by the method of Aiba et al. (1981) with a minor modification. T. aquaticus NTU103 were cultured for different periods of time and the cell pellets were washed with TE [10 mM Tris-Cl, $1 \mathrm{mM}$ EDTA (pH 8.0)] buffer. After the pellets were suspended in $2 \mathrm{ml}$ RNA extraction buffer ( $25 \mathrm{mM}$ EDTA, 10\% sucrose, 
$25 \mathrm{mM}$ Tris-Cl, $\mathrm{pH}$ 8.0, and RNase free DNase $20 \mathrm{U}$ ) and incubated on ice for $15 \mathrm{~min}$. RNA was extracted twice with equal volumes of acidified $60^{\circ} \mathrm{C}$ phenol. RNA was precipitated with ice-cold ethanol following chloroform extraction. Reverse transcriptase-polymerase chain reaction was performed with primers, rep5' $\left(5^{\prime}\right.$-ATGGGCTA CCGGGTGGCCCAT) and rep3'(5'-ATCGGTGAGCA CCCCTTCGGA) for rep gene and ORF2-5' $\left(5^{\prime}\right.$-AT GGGCCACACGCTTCACAGC) and ORF2-3' (5'-GGC TATCCTGGGGGTGCCCCC) for rep gene and orf2, respectively. Northern blotting was conducted with DIGlabeled gene-specific probes of rep and orf2. The rep genespecific probe for Northern blotting was generated with primers rep5'-2(5'-TATTGGCCGGGCGTTCGGGG) and rep3'. Meanwhile, orf2 gene-specific probe was synthesize by ORF2-5'-2(5'-GCCTTAGCCAGAATCTCG CCC) and ORF2-3' by polymerase chain reaction. RNase-treated samples were prepared as a negative control.

\section{4. ssDNA detection and southern hybridization}

Bacteria were harvested in exponential phase after culturing in Thermus medium and total DNA was extracted as previously described (Kado and Liu, 1981). ssDNA was detected according to the method described elsewhere (Noirot-Gros and Ehrlich, 1996), with or without S1 nuclease treatment and with or without DNA denaturation prior the gel blotting. Southern hybridization was performed following the instructions of manufacturer of the DIG-High Prime Kit (Roche, Taipei, Taiwan).

\subsection{Nucleotide sequences accession number}

The GenBank accession number for pTA103 is AY190303.

\section{Results and discussion}

\subsection{Hybridization of pTA103 and the plasmids in $T$. aquacticus YT-1}

Thermus aquacticus NTU103 contained two cryptic plasmids, of which pTA103 is the smaller one, and was studied herein. Sequence analysis of 16S rRNA gene showed that NTU103 shared 99.8\% similarity with $T$. aquaticus YT-1 (ATCC25104), isolated from Yellow Stone National Park in the United States (Brock and Freeze, 1969). Plasmids from T. aquacticus YT-1 and NTU103 were analyzed by $0.8 \%$ agarose gel electrophoresis followed by southern hybridization with DIG-labeled pTA103 as a probe. The results indicated that these two strains included no plasmids with a similar size (Fig. 1. lanes 1 and 2).

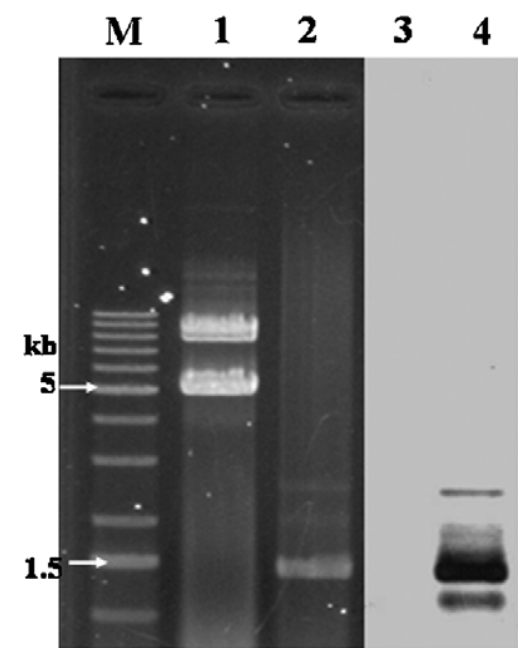

Fig. 1. Southern hybridization of pTA103 and plasmids from $T$. aquaticus YT-1. Plasmids were separated by agarose gel electrophoresis (lanes 1and 2) and Southern blot analysis (lanes 3 and 4), using pTA103 as a probe. Lanes 1 and 3, plasmids from Thermus aqauticus YT-1 and, lanes 2 and 4, plasmids from T. aqaticus NTU103.

Southern hybridization also revealed that plasmids in T. aquaticus YT-1 shared no homology with pTA103. (Fig. 1. lanes 3 and 4). These results also indicated that although the $16 \mathrm{~S}$ rRNA gene in T. aquaticus NTU103 and YT-1 were 99\% similar, the plasmid profiles of these two strains were quite distinct and the plasmids in these two strains shared no sequence homology.

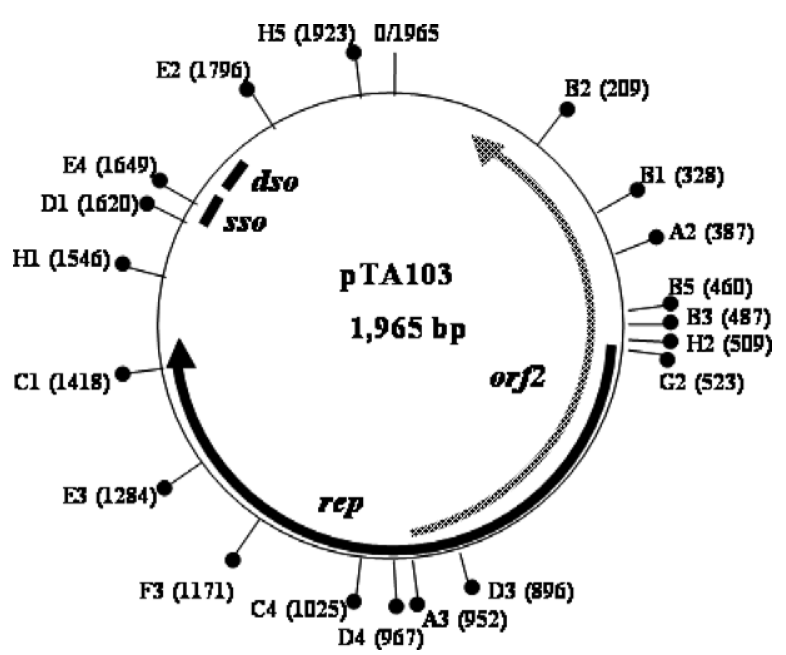

Fig. 2. Map and transposon insertions in pTA103. rep and orf2 are two open reading frames in transcribed from opposite directions. Black circles indicate the locations of transposon insertions. Putative sso and $d s o$ were located downstream of rep gene. Numbers denote the nucleotide positions. 


\subsection{DNA sequences analysis of $p T A 103$}

Sequences of pTA103 were determined by using primers near the borders of EN::TN $\langle$ R6K $\gamma$ ori KAN2) (Fig. 2). Sequencing results demonstrated that pTA103 is $1965 \mathrm{bp}$ long and has a $\mathrm{G}+\mathrm{C}$ content as high as $67.5 \%$, which was similar to chromosome $\mathrm{G}+\mathrm{C}$ content $(65.4-67.4 \%)$ of $T$. aquaticus YT-1 (Brock and Freeze, 1969). Sequence analysis also revealed that pTA103 contained at least two open reading frames; one of which, orf 2 (from nt 947 to nt 120) (Fig. 2) shared no homology with any protein from databases. The other orf (from nt 513 to nt 1454) (Fig. 2) shared 50\% similarity and 34\% identity with the Rep-like protein of $\mathrm{pRm} 1132 \mathrm{f}$, which is a group III RCR plasmid (Barran et al., 2001).

As is generally known, RCR plasmids are usually classified based on sequence homologies among their Rep-like proteins and $d s o$ s. The structure of Rep-like proteins contained at least two domains: one involved in origin nicking; the other, specific recognition of a $d s o$ sequence (Khan, 2005). All Replike proteins belonging to a particular family shared highly conserved nicking domain, which contain a conserved tyrosine residue (del Solar et al., 1998; Ilyina and Koonin, 1992; Khan, 1997; Projan and Novick, 1988). This tyrosine residue is located at position 121 of the Rep-like protein encoded by pTA103 (Fig. 3A). On the other hand, the DNAbinding domain in the pTA103 Rep protein differs from that of the other Rep proteins (Projan and Novick, 1988). According to the phylogenetic analysis of the pTA103 Rep-like protein, the protein likely belongs to the third family of the RCR plasmids. Meanwhile, pTA103 is the first reported RCR plasmid in T. aquaticus.

\section{3. dso and sso}

Most RCR plasmids are relatively small, ranging from 0.8 to $10 \mathrm{~kb}$. Khan (1997) classified these plasmids into five groups, based on the homology in their initiator Rep proteins and leading or dsos. Replication of the leading-strand of RCR plasmids initiated from the $d s o$. The sequences of $d s o$ s usually have a secondary structure, either within or upstream of the rep gene (Khan, 1997). Although the Rep-like protein of pTA103 shared a 50\% similarity with a group III Rep protein, a sequence that is homologous to the group III $d s o$ s has not been found upstream of the gene that encodes the Rep-like protein.

Unlike the $d s o$ sequences, the sso sequences among plasmids of the same family are generally unrelated. Several types of $s s o$, such as $\operatorname{sso} A$, $s s o U$, ssoT, and sso $W$ have been identified according to their secondary structures and sequence homology (Andrup et al., 1994; Boe et al., 1989; del Solar et al., 1993; Kramer et al., 1995, 1999; Madsen et al., 1993; Meijer et al., 1995; Seeger et al., 1995; Zaman et al., 1993). Khan (1997) posited that sso generally locates
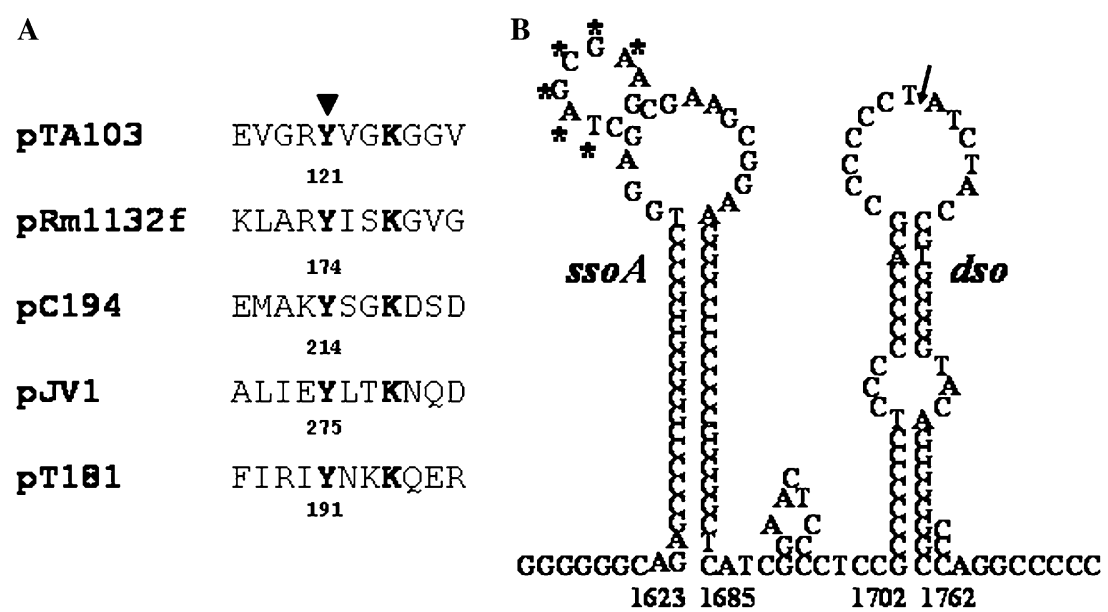

Fig. 3. Sequence alignment of active site of Rep-like proteins and secondary structure analysis of the sequence between the nt 1675 and the nt 1770 region in pTA103. (A) Amino acid sequence alignment of the active site of Rep-like proteins of five RCR plasmids. Tyrosine residue involving in nicking plasmid DNA is indicated with a triangle. The numbers denote the location of the tyrosine residue in the active site in Rep-like proteins. (B) Secondary structures of putative $s s o A$ and $d s o$ of pTA103. Asterisks mark the $\mathrm{CS}_{6}$ of $s s o A$. Arrow indicates the nic site of a putative dso. 
at a short distance upstream of $d s o$. Like the $d s o$, ssos also contain a sequence that forms a secondary structure. RCR plasmids with sso $A$ origins exhibit a conserved six-nucleotide sequence, 5'-TAGCGt/a-3'. Plasmid pTA103 also contains a similar sequence of 5'-TAGCGA-3 located in a 62-bp palindromic structure between nt 1623 and nt 1685 (Fig. 3B), which potentially forms a hairpin structure. Accordingly, another palindromic structure with a length of $61 \mathrm{bp}$ located between nt 1702 and nt 1762 was found downstream of the putative $s s o A$. This region may function as the $d s o$ for pTA103 RC replication. The possible nic site is likely at nt 1728. (Fig. 3B).

\subsection{Detection of ssDNA}

The single-stranded DNA (ssDNA) intermediates from the exponential-phase cells were analyzed to verify that pTA103 replicates with the RCR mechanism. ssDNA is detected according to its $\mathrm{S} 1$ nuclease sensitivity and nitrocellulose-binding property. Southern hybridization detected ssDNA that hybridized to DIG-labeled pTA103 probe (Fig. 4, lane 1). On the other hand, the DNA was undetected if the sample was treated with S1 nucleases (Fig. 4, lanes 2-4). Double-stranded DNA was applied to electrophoresis and was alkaline denatured before blotting served as positive control (Fig. 4, lane 5). Without the alkaline treatment, double-stranded DNA does not bind to nitrocellulose filter. This clearly suggests the existence of pTA103 ssDNA intermediates in T. aquaticus NTU103. This result is the direct evidence that pTA103 is an RCR plasmid.

\subsection{Detecting RNA transcripts}

Thermus aquaticus NTU103 was cultured in $50 \mathrm{~mL}$ of Thermus medium and bacteria were

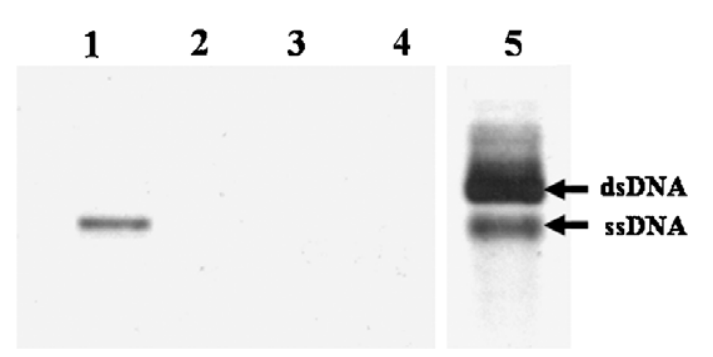

Fig. 4. S1 nuclease analysis of pTA103. Lane 1, plasmid sample untreated with S1 nuclease; lanes 2-4, DNA treated with S1 nuclease for 20,30 , and $60 \mathrm{~min}$, respectively; lane 5, pTA103 denatured by alkaline buffer before Southern blotting. collected in the middle exponential phase and stationary phase. RT-PCR was performed with MMLV reverse transcriptase at $37^{\circ} \mathrm{C}$. The RT-PCR products of rep and orf 2 were amplified using their specific primers. The results revealed that both open reading frames were amplified with different size. (Fig. 5A, lanes 2 and 5). An amplification with RNase-treated samples was used as a negative control (Fig. 5A, lanes 1 and 4); amplification with pTA103 as template served as positive controls (Fig. 5A, lanes 3 and 6). Additionally, Northern blotting showed that the transcripts of rep-like gene and $\operatorname{orf} 2$ could be detected simultaneously under different growth condition (Fig. 5B, lanes 1 and 2). The transcript of $\operatorname{orf} 2$ was observed to be a shorter transcript (Fig. 5B, lanes 3 and 4). Both experiments demonstrated that these two open reading frames express transcripts at the middle exponential phase (Fig. 5B. Lane 1,3) and stationary phase (Fig. 5B. Lane 2,4). Because plasmid replication may be controlled by antisense RNA (del Solar et al., 1998), whether the replication of pTA103 is affected by orf2 is of interest. In pTA103, the rep and orf 2 open reading frames overlap and are transcribed from the opposite orientations. This phenomenon was also observed for the two open reading frames in pRm1132 (Barran et al., 2001). Further investigation must be conducted for elucidating the function of orf2 on the opposite transcription orientation of rep-like gene.

This report characterizes a RCR plasmid, pTA103 from $T$. aquaticus NTU103. The possible functional regions of $d s o$ and $s S O A$ for this plasmid are predicted.
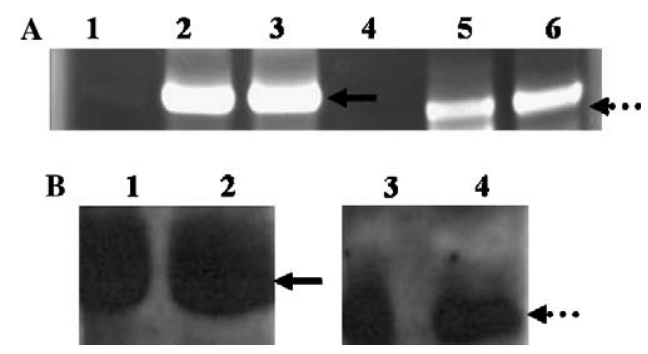

Fig. 5. RNA transcripts from rep and orf 2. (A) Transcripts were analyzed using RT-PCR with specific primers for each open reading frame. Lanes 1-3 are the products of the rep transcript. Lanes 4-6 are the products of orf 2. (B) Northern hybridization of the rep and orf 2 transcripts. Lanes 1 and 2 are transcripts hybridized with a rep-specific probe. Lanes 3 and 4 are transcripts hybridized using an orf2-specific probe. Arrow indicates the transcript of the rep-like gene. Dashed arrow denotes the transcript of $\operatorname{orf} 2$. 


\section{Acknowledgments}

The authors thank the National Science Council of the Republic of China, Taiwan for financially supporting this research under Contract No NSC932320-B-320-006. This work was also supported by Tzu-Chi University under Contract No. TCMRC93006.

\section{References}

Aiba, H., Adhya, S., de Crombrugghe, B., 1981. Evidence for two functional gal promoter in intact Escherichia coli cells. J. Biol. Chem. 256, 11910-11950.

Andrup, L., Dammgaard, J., Wasserman, K., Boe, L., Madsen, S.M., Hansen, F.G., 1994. Complete nucleotide sequence of the Bacillus thuringiensis subsp. israelensis plasmid pTX14-3 and its correlation with biological properties. Plasmid 31, $72-88$.

Barran, L.R., Ritchot, N., Bromfield, E.S., 2001. Sinorhizobium meliloti plasmid $\mathrm{pRm} 1132 \mathrm{f}$ replicates by a rolling-circle mechanism. J. Bacteriol. 183, 2704-2708.

Birnboim, H.C., Doly, J., 1979. Rapid alkaline extraction procedure for screening recombinant plasmid DNA. Nucleic Acids Res. 7, 1513-1523.

Boe, L., Gros, M.F., te Riele, H., Ehrlich, S.D., Gruss, A., 1989. Replication origins of single-stranded-DNA plasmid pUB110. J. Bacteriol. 171, 3366-3372.

Brock, T.D., Freeze, H., 1969. Thermus aquaticus gen. n. and sp. n., a nonsporulating extreme thermophile. J. Bacteriol. 98, 289-297.

del Solar, G., Kramer, M.G., Ballester, S., Espinosa, M., 1993. Replication of the promiscuous plasmid pLS1: a region encompassing the minus origin of replication is associated with stable plasmid inheritance. Mol. Gen. Genet. 241, 97-105.

del Solar, G., Giraldo, R., Ruiz-Echevarria, M.J., Espinosa, M., Diaz-Orejas, R., 1998. Replication and control of circular bacterial plasmids. Microbiol. Mol. Biol. Rev. 62, 434-464.

Erauso, G., Marsin, S., Benbouzid-Rollet, N., Baucher, M.F., Barbeyron, T., Zivanovic, Y., Prieur, D., Forterre, P., 1996. Sequence of plasmid pGT5 from the archaeon Pyrococcus abyssi: evidence for rolling circle replication in a hyperthermophile. J. Bacteriol. 178, 3232-3237.

Goryshin, I.Y., Reznikoff, W.S., 1998. Tn5 in vitro transposition. J. Biol. Chem. 273, 7367-7374.

Gruss, A., Ehrlich, S.D., 1989. The family of highly interrelated single-stranded dexoyribionucleic acid plasmids. Micrbiol. Rev. 53, 231-241.

Harriott, O.T., Huber, R., Stetter, K.O., Betts, P.W., Noll, K.M., 1994. A cryptic miniplasmid from the hyperthermophilic bacterium Thermotoga sp. strain RQ7. J. Bacteriol. 176, 2759-2762.

Hishinuma, F., Tanaka, T., Sakaguchi, K., 1978. Isolation of extrachromosomal deoxyribonucleic acids from extremely thermophilic bacteria. J. Gen. Microbiol. 104, 193-199.

Ilyina, T.V., Koonin, E.V., 1992. Conserved sequence motifs in the initiator proteins for rolling circle DNA replication encoded by diverse replicons from eubacteria, eukaryotes and archaebacteria. Nucleic Acid Res. 20, 3279-3285.

Jonuscheit, M., Martusewitsch, E., Stedman, K.M., Schleper, C., 2003. A reporter gene system for the hyperthermophilic archa- eon Sulfolobus solfataricus based on a selectable and integrative shuttle vector. Mol. Microbiol. 48, 1241-1252.

Kado, C.I., Liu, S.T., 1981. Rapid procedure for detection and isolation of large and small plasmid. J. Bacteriol. 145, 1366-1373.

Khan, S.A., 1997. Rolling-circle replication of bacterial plasmids. Microbiol. Mol. Biol. Rev. 61, 442-455.

Khan, S.A., 2005. Plasmid rolling-circle replication: highlights of two decades of research. Plasmid 53, 126-136.

Kleanthous, H., Clayton, C.L., Tabaqchali, S., 1991. Characterization of a plasmid from Helicobacter pylori encoding a replication protein common to plasmids in gram-positive bacteria. Mol. Microbiol. 5, 2377-2389.

Kramer, M.G., del Solar, G., Espinosa, M., 1995. Laggingstand origins of the promiscuous plasmid pMV158: physical and functional characterization. Microbiology 141, 655-662.

Kramer, M.G., Espinosa, M., Misra, T.K., Khan, S.A., 1999. Characterization of a single-stand origin, sso $U$, required for broad host range replication of rolling-circle plasmids. Mol. Microbiol. 33, 466-475.

Kues, U., Stahl, U., 1989. Replication of plasmids in gram-negative bacteria. Microbiol. Rev. 53, 491-516.

Lasa, I., de Grado, M., de Pedro, M.A., Berenguer, J., 1992. Development of Thermus-Escherichia shuttle vectors and their use for expression of the Clostridium thermocellum celA gene in Thermus thermophilus. J. Bacteriol. 174, 6424-6431.

Madsen, S.M., Andrup, L., Boe, L., 1993. Fine mapping and DNA sequence of replication functions of Bacillus thuringiensis plasmid pTX14-3. Plasmid 30, 119-130.

Mather, M.M., Fee, J.A., 1990. Plasmid-associated aggregation in "Thermus thermophilus" HB-8. Plasmid 24, 45-56.

Meijer, W.J., Venema, G., Bron, S., 1995. Characterization of single stand origins of cryptic rolling-circle plasmids from Bacillus subtilis. Nucleic Acids Res. 23, 612-619.

Noirot-Gros, M.F., Ehrlich, S.D., 1996. Change of a catalytic reaction carried out by a DNA replication protein. Science 274, 777-780.

Projan, S.J., Novick, R.P., 1988. Comparative analysis of five related staphylococcal plasmids. Plasmid 19, 203-221.

Seeger, J.F., Zhao, A.C., Meijer, W.J., Khan, S.A., Venema, G., Bron, S., 1995. Structural and functional analysis of the singlestrand origin of replication from lactococcal plasmid $\mathrm{pWVO1.}$ Mol. Gen. Genet. 249, 43-50.

Tabata, K., Ishida, S., Nakahara, T., Hoshino, T., 1994. A carotenogenic gene cluster exists on a large plasmid in Thermus thermophilus. FEBS Lett. 341, 251-255.

Vásquez, C., Villanueva, J., Vicuňa, R., 1983. Plasmid curing in "Thermus thermophilus" and "Thermus flavus". FEBS Lett. 158, 339-342.

Wayne, J., Xu, S.Y., 1997. Identification of a thermophilic plasmid origin and its cloning within a new Thermus-E. coli shuttle vector. Gene 195, 321-328.

Williams, R.A.D., da Costa, M.S., 1992. The genus Thermus and related microorganism. In: Balows, A., Truper, H., Dworkin, G., Harder, M.W., Schleifer, K.-H. (Eds.), The Prokaryotes, second edn. Springer, New York, pp. 3745-3753.

Yasukawa, H., Hase, T., Sakai, A., Masamune, Y., 1991. Rolling-circle replication of the plasmid $\mathrm{pKYM}$ isolated from a gram-negative bacterium. Proc. Natl. Acad. Sci. USA 88, 10282-10286. 
York, D., Welch, K., Goryshin, I.Y., Reznikoff, W.S., 1998. Simple and efficient generation in vitro of nested deletions and inversions: Tn5 intramolecular transposition. Nucleic Acids Res. 26, 1927-1933.

Yu, J.S., Noll, K.M., 1997. Plasmid pRQ7 from the hyperthermophilic bacterium Thermotoga species strain RQ7 replicates by the rolling-circle mechanism. J. Bacteriol. 179, 7161-7164.

Zaman, S., Radnedge, L., Richards, H., Ward, J.M., 1993. Analysis of the site for second-strand initiation during replication of the Streptomyces plasmid pIJ101. J. Gen. Microbiol. 139, 669-676. 